\title{
Fuzzy Damage-Mitigating Control of a Fossil Power Plant
}

\author{
Michael Holmes and Asok Ray, Senior Member, IEEE
}

\begin{abstract}
This paper presents the architecture and synthesis of a damage-mitigating control system where the objective is to achieve high performance with increased reliability, availability, component durability, and maintainability. The proposed control system has a two-tier structure. In the lower tier, a linear robust sampled-data controller tracks a reference trajectory vector while the upper tier contains a fuzzy-logic-based damage controller that makes a tradeoff between system dynamic performance and structural durability in critical component(s). The synthesis procedure is demonstrated on the model of a commercial-scale fossil-fueled power plant under load-following operation. Simulation experiments are designed to explore the feasibility of real-time fuzzy damage-mitigating control in fossil power plants, and the results show that substantial gain in structural durability of a critical component can be achieved with no significant loss of performance.
\end{abstract}

Index Terms-Fatigue damage mitigation, fuzzy control, power plant control, robust control, sampled-data control.

\section{INTRODUCTION}

$\mathbf{O}$ PERATION and control procedures for fossil power plants require decision-making based on tradeoff between performance enhancement and life extension [10]. The current state-of-the-art of synthesizing power plant control systems focuses on guaranteeing stability of the closed loop system while simultaneously ensuring that the specified performance requirements are satisfied. The measure of power plant performance is usually expressed in terms of 1) thermodynamic efficiency under steady-state operations and 2) reference signal tracking (e.g., matching of actual generated power with the load demand), disturbance rejection, and/or control effort minimization under transient operations. However, performance specifications do not explicitly address the time-dependent behavior of material damage (e.g., fatigue cracking and creep) in critical plant components. This paper introduces a concept of fuzzy damage-mitigating control for life extension of fossil power plants with no significant reduction in performance.

Damage mitigating control is a relatively new area of research that combines two distinct disciplines: Systems Sciences and Mechanics of Materials. Currently, there is a rather limited amount of information available on this topic in the literature. [9] and [3] have shown that, in an open loop setting, it is feasible

Manuscript received December 9, 1998; revised March 7, 2000 and July 27, 2000. Recommended by Associate Editor A. Stankovic. This work was supported in part by the National Science Foundation under Research Grants CMS-9819074 and ECS-9912495 and Electric Power Research Institute Contract EPRI RP8030-05.

The authors are with the Mechanical Engineering Department, The Pennsylvania State University, University Park, PA 16802 USA (e-mail: axr2@ psu.edu). Publisher Item Identifier S 1063-6536(01)00526-7. to reduce the damage rate and accumulation in critical components of a reusable rocket engine without any significant sacrifice of the plant performance. Their damage reduction procedure, however, is based on an extensive off-line optimization and does not take advantage of on-line damage predictions or measurements. Also, the resulting feedforward signal is optimized for a particular set of initial conditions and a maneuver that must be specified a priori. Kallappa and Ray [5], [6] have proposed a feedforward-feedback methodology for the synthesis of robust linear control systems for life extension of fossil-fueled power plants. Holmes and Ray [4] have used a similar approach for life-extending control of reusable rocket engines via output feedback. Zhang and Ray [13] have demonstrated this control concept on a laboratory testbed to achieve a tradeoff between dynamic performance and structural durability.

This paper presents a procedure for synthesizing a damage mitigating control system for fossil-fueled power plants that has a two-tier structure. In the lower tier a linear sampled-data controller tracks a reference trajectory vector while the upper tier contains a fuzzy-logic-based damage controller which makes a tradeoff between the plant dynamic performance and the damage in critical plant components. The synthesis procedure is tested by simulation experiments on the model of a commercial-scale fossil power plant [11]. The objective here is to explore the feasibility of automatically regulating the damage/performance tradeoff in a real-time setting based on on-line damage rate calculations. A power plant has many critical components such as steam generators, main steam and hot reheat steam headers, and main and boiler feedpump turbines, and pumps. All these critical components must be taken into consideration in the synthesis of a damage-mitigating control system before its implementation in an operating power plant. To elucidate the underlying principle of damage-mitigating control, this paper focuses on fatigue crack damage in a specific critical component of fossil power plants, namely, the main steam header that feeds superheated steam from main steam generators into high pressure turbines.

\section{Modeling of Plant Dynamics and Structural} DAMAGE

The power plant under consideration is a commercial-scale once-through fossil unit having a rated generation capacity of $525 \mathrm{MWe}$. The plant maintains the throttle steam condition at $950^{\circ} \mathrm{F}\left(510^{\circ} \mathrm{C}\right)$ and $2415 \mathrm{psia}(16.65 \mathrm{Mpa})$ and the hot reheat steam temperature at $1000^{\circ} \mathrm{F}\left(537.8^{\circ} \mathrm{C}\right)$. The (finite-dimensional) dynamic model of the power plant was formulated as a set of coupled nonlinear time-invariant differential equations via 
lumped parameter approximation of the governing partial differential equations. The plant dynamic model has 27 state variables, four control inputs and four output variables. Four valves, namely, the turbine governor valve, the fuel/air valve, the feedpump turbine valve, and the reheat attemperator valve, are selected as the control actuators. The measured output variables used in the control system are: throttle steam temperature; hot reheat steam temperature; throttle steam pressure; and electric power. Details of the model equations are reported by Weng et al. [11].

The damage prediction model, which is an essential ingredient of the damage-mitigating control system, is coupled to the structural model of a critical plant component. Selected plant outputs serve as inputs to the structural model that generates necessary information for the damage prediction model. The output of the structural model is the structural stress vector which, for example, may consist of time-dependent stresses, strains, and temperatures at critical points of the structure (e.g., the main steam header in a power plant). The damage state vector quantifies structural degradation, for example, due to fatigue cracking and creep. The time derivative of the damage vector indicates how the instantaneous load is affecting the critical plant component which is the main steam header in this paper. Damage modeling of other plant components (e.g., steam generator tubes) has been reported in [6].

\section{Damage Mitigating Control Architecture}

A schematic diagram of the fuzzy damage-mitigating control system for the fossil power plant is shown in Fig. 1 where the plant has three types of sensor outputs: $y^{\mathrm{dam}}(t), y^{\mathrm{dyn}}(t)$, and $y^{\mathrm{reg}}(t)$. The vector signal $y^{\mathrm{dam}}(t)$ contains those plant outputs that are necessary for the calculation of damage (e.g., throttle steam temperature, throttle steam pressure and steam flow rate through the main steam header for the calculation of fatigue and creep damage in the main steam header wall). The vector signal $y^{\text {dyn }}(t)$ consists of the plant outputs whose reference trajectory vector is determined by the damage controller (e.g., throttle steam temperature in the power plant). The rationale for choosing the throttle steam temperature as the plant output reference signal being determined by the fuzzy controller is discussed in Section V. The vector signal $y^{\mathrm{reg}}(t)$ contains regulated plant outputs whose reference signals are either constants or unaltered by the damage controller. The vector signal $y^{\mathrm{reg}}(t)$ consists of throttle steam pressure, hot reheat steam temperature, and electric power.

The purpose of the linear tracking controller in Fig. 1 is to keep the error signals as close to zero as possible, i.e., to track $y^{\text {set }}(k)$ and $y^{\mathrm{ref}}(k)$, and to provide robust stability in the inner (linear) control loop. Details on the design of the linear controller are presented in Section III. Unlike the work in [12] and [5], the feedforward control input, $u^{\mathrm{ff}}(k)$, is not an optimized sequence and is actually a function of the electric power reference trajectory which is a component of the vector signal $y^{\text {set }}(t)$. The relationship is selected as $u^{\mathrm{ff}}(k)=c_{1} y_{\mathrm{JGN}}^{\mathrm{set}}(k)+c_{2}$, where the two constants, $c_{1}$ and $c_{2}$, are identified $a$ priori based on the linear interpolation of steady-state inputs and $y_{\mathrm{JGN}}^{\mathrm{set}}(k)$ is the electric power reference trajectory.

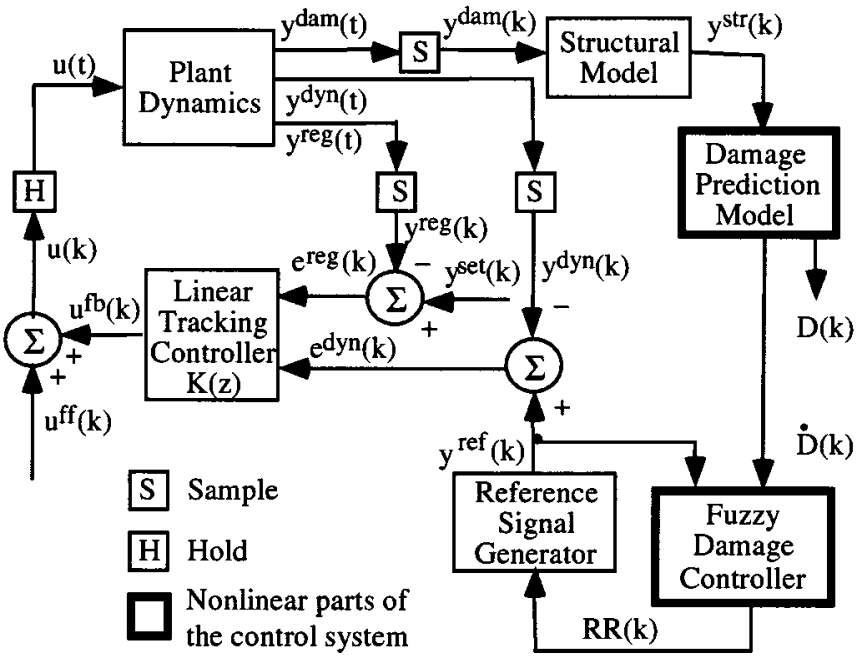

Fig. 1. Schematic diagram of fuzzy damage-mitigating control.

The structural model in Fig. 1 uses the vector $y^{\text {dam }}(k)$ as an input to generate damage-causing variables such as stresses, $y^{\operatorname{str}}(k)$. The signal $y^{\operatorname{str}}(k)$ excites the damage model whose output is both damage rate and damage accumulation. The purpose of the damage model in the outer control loop is to capture the dynamic characteristics of material degradation under stress. The critical component being considered for the power plant is the fatigue damage occurring in the wall of the main steam header. The damage model is highly nonlinear and is normalized to have an output in the range $[0,1]$ where a value of zero can be interpreted as the state of material defects in a new header and a value of one implies that the service life of the component has been expended. The damage model used in the damage feedback loop provides a measure of damage rate to the damage controller. Since damage is not reversible, the damage rate is always a nonnegative quantity.

The reference signal generator takes the ramp rate specified by the damage controller and integrates it to obtain the reference signal. The output of the integrator is the input of a saturation function which limits the throttle steam temperature reference signal to lie within the range $940^{\circ} \mathrm{F}$ to $950{ }^{\circ} \mathrm{F}$. In this application, the fuzzy damage controller alters the ramp rate of the reference trajectory of throttle steam temperature as a function of the current value of damage rate. Based on the operational constraints of the plant, the output of the fuzzy damage controller is constrained within the prespecified range of $R R_{\min } \leq$ $R R(k) \leq R R_{\max }$.

\section{LinEAR RobuSt SAMPLED-DATA TRACKING CONTROLLER}

In this section the sampled-data tracking controller used in the fuzzy damage mitigation architecture shown in Fig. 1 is designed for the fossil power plant by using the $H_{\infty}$ (or induced $L_{2}$-norm to $L_{2}$-norm) controller synthesis technique. Bamieh and Pearson [2] proposed a solution to the induced $L_{2}$-norm controller synthesis problem for application to sampled-data systems. The $D-K$ iteration technique is also used where "suboptimal" rational polynomial weights ( $D$ 's) are found using $\mu$-synthesis and the controller $(K)$ is found using 


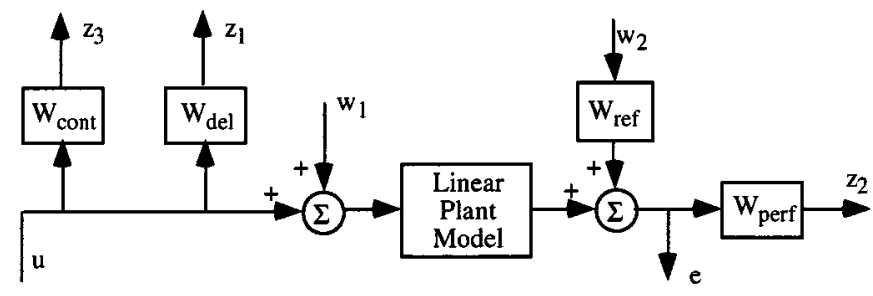

Fig. 2. The generalized plant model for linear robust controller synthesis.

the induced $L_{2}$ sampled-data design procedure. The $D$ 's are suboptimal because they are obtained from $\mu$-synthesis based on the $H_{\infty}$ norm of the discretized closed-loop system (i.e., a discrete controller connected to the discretized generalized plant), which is not equivalent to the induced $L_{2}$ norm of the closed-loop sampled-data system.

The linear plant model in Fig. 2 is obtained by linearizing the 27-state nonlinear model of the power plant which is briefly described in Section II. The linearization takes place at the steady state at which the throttle temperature is $950^{\circ} \mathrm{F}$, the hot reheat steam temperature is $1000^{\circ} \mathrm{F}$, the throttle pressure is 2415 psia, and the electrical power is $367.5 \mathrm{MW}$ (70\% load). The electrical power of $367.5 \mathrm{MW}$ ( $70 \%$ load) is chosen for linearization because the controller is required to operate within the range $210 \mathrm{MW}$ (40\% load) to $525 \mathrm{MW}$ (100\% load). The modeling error inherent in the linear plant model used for the design is represented by input multiplicative modeling uncertainty. The weighting matrix chosen for the uncertainty description is the identity matrix multiplied by the strictly proper function

$$
W_{\text {del }}(s)=2\left(\frac{s+1}{s+20}\right)\left(\frac{1.0 \times 10^{5}}{s+1.0 \times 10^{5}}\right)
$$

which implies that the amount of plant uncertainty is estimated as being $10 \%$ at low frequencies and $200 \%$ at high frequencies. The extra high frequency dynamics in (1) are necessary to ensure that the generalized plant has a zero $D$ matrix as is required for the use of the MATLAB function sdhfsyn.

The reference signal weighting matrix is chosen to be the identity matrix multiplied by the strictly proper function

$$
W_{\text {ref }}(s)=\frac{0.005}{s+0.005}
$$

which implies that disturbances with frequency content of less than $0.005 \mathrm{rad} / \mathrm{s}$ are expected.

In this application, weighting the four error signals with weights containing no dynamics is found to produce a controller with satisfactory performance. The frequency-dependent performance weight in Fig. 2 is chosen as

$$
W_{\text {perf }}(s)=\operatorname{diag}\{230,230,20,60\}
$$

where the order of outputs is the same as listed above. The first two entries in $W_{\text {perf }}$ that penalize throttle steam temperature and hot reheat steam temperature most heavily as to reduce temperature transients that cause thermal stresses leading to thermo-mechanical fatigue. The third entry that penalizes throttle steam pressure is weighted the least as pressure transients have relatively less sever effects on structural durability of plant components. The last entry penalizes electrical power to reduce devia- tions of the generated power from its desired profile. Hence, the linear robust controller partially plays the role of damage mitigation by penalizing the damage-causing variables. The fuzzy controller achieves additional damage compensation as explained later in Section V.

The four controller output signals tend to be small because of the presence of the plant model uncertainty weight, $W_{\text {del }}(s)$. However, it was found that the fourth plant input (i.e., the attemperator valve area) saturates during some simulations unless the fourth controller output is included as an additional output of the generalized plant. The control signal weight, $W_{\text {cont }}(s)$, in Fig. 2 is only being used for the fourth control signal and is chosen to be

$$
W_{\text {cont }}(s)=5000\left(\frac{1.0 \times 10^{5}}{s+1.0 \times 10^{\tilde{5}}}\right)
$$

where, again, the extra high frequency dynamics are necessary to ensure that the generalized plant model is strictly proper.

The linear feedback controller is designed by using MATLAB mutools toolbox [1] based on the method outlined above. Five $D-K$ iterations are performed where the order of the fits for the $D$ 's are chosen to be five, five, two one, and three. This procedure produces a robustly stable digital controller having 47 states. The controller contains such a large number of states because the synthesis method produces a digital controller with the same number of states as the generalized plant. The generalized plant, in this case, contains the dynamics of the linear plant (27 states), the control signal weighting function (one state), the reference signal weighting matrix (four states), the input multiplicative plant uncertainty weighting matrix (eight states), and the dynamics of the weighting functions used in the next to last iteration of the $\mu$-synthesis procedure (i.e., the $D$ 's mentioned above) (eight states). In addition, the generalized plant is balanced before it is used for controller synthesis. This balancing procedure automatically removes one state $(-1$ state) without making the generalized plant's $D$ matrix nonzero. Therefore, all 47 of the controller states are accounted for. After applying Hankel model reduction to this controller, a 13 state robustly stable controller is obtained which suffers very little performance loss when compared to the 47 state controller.

\section{FUZZY CONTROLLER SYNTHESIS FOR DAMAGE-MitigATION}

The proposed procedure for synthesis of a fuzzy controller for damage mitigation in critical plant component(s) consists of the following three steps:

- Knowledge acquisition that involves: 1) understanding of the operation and dynamics of the underlying physics of the plant; 2) identification of those plant outputs which directly influence damage occurring in the critical components under consideration; and 3) characterization of the specific behavior of the plant outputs that causes large damage rates in the critical plant components.

- Formulation of a structure for the fuzzy controller which involves: 1) selection of fuzzy controller inputs (i.e., the quantities a human operator would need to see while performing damage-mitigating control); 2) selection of fuzzy controller outputs (i.e., the quantities a human 


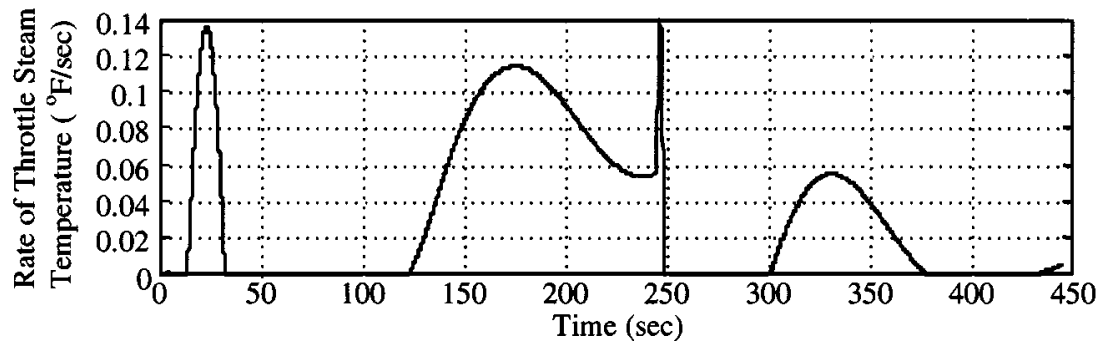

Fig. 3. Throttle steam temperature rate.

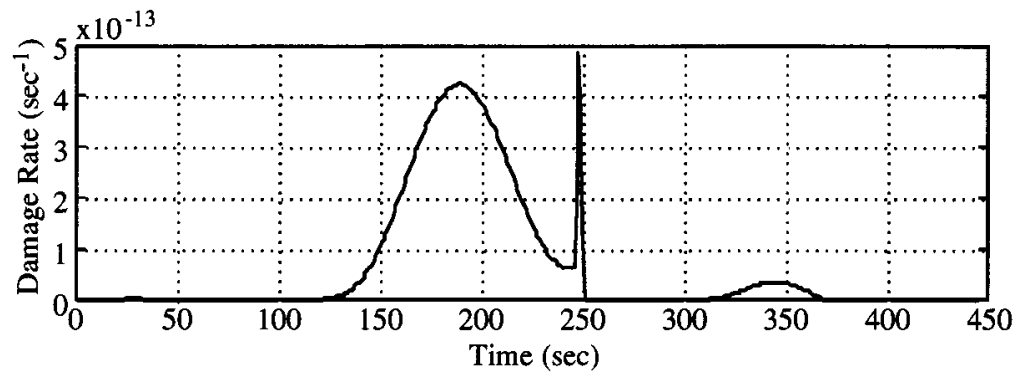

Fig. 4. Fatigue damage rate.

operator would alter while performing damage-mitigating control); and 3) construction of an internal structure for the fuzzy controller (i.e., an initial choice of the membership functions and if-then rules based on the human operator's knowledge).

- Adjustment of the parameters in the fuzzy controller to fine-tune the controller performance.

The plant outputs that are necessary for the calculation of fatigue damage (e.g., the length of the cracks in the outer wall of the main steam header) are throttle steam temperature, throttle steam pressure, and steam flow rate. The steam flow rate has only a minimal effect on the damage rate, whereas there is a high correlation between the damage rate and the time derivative of throttle steam temperature. Fig. 3 contains a plot of the derivative of the throttle steam temperature for a typical power plant maneuver after being filtered with a saturation function

$$
\dot{y}_{\mathrm{sat}}^{\mathrm{THS}}(t)= \begin{cases}\dot{y}^{\mathrm{THS}}(t) & \text { if } \dot{y}^{\mathrm{THS}}(t)>0 \\ 0 & \text { if } \dot{y}^{\mathrm{THS}}(t) \leq 0\end{cases}
$$

where $y^{\text {THS }}(t)$ is the throttle steam temperature and $\dot{y}^{\text {THS }}(t)$ is its time derivative. Fig. 4 shows the fatigue damage rate for this same maneuver. Although there are other factors (e.g., the throttle steam pressure and steam flow rate) that influence fatigue damage in the main steam header, both plant experience and finite-element analysis show that the fatigue damage rate is strongly dependent on the throttle steam temperature rate when this rate is positive. Figs. 3 and 4 indicate that if the derivative of the throttle steam temperature rate is high, then the fatigue damage rate is high and that the resulting time delay in the fatigue rate response is due to the presence of dynamics in the structural and damage models. The spike in the temperature rate plot of Fig. 3 at around the instant of $250 \mathrm{~s}$ results from an abrupt decrease in the rate of governor valve opening which closely follows the power reference trajectory as commanded by the linear tracking controller. This, in turn, causes a sharp pulse in the steam pressure rate and therefore the steam temperature rate.

A knowledge base of structural damage is generated from a combination of a variety of computer simulations and the available information on actual plant operating experience. Both plant operating experience and simulation experiments reveal that if the fatigue damage rate is high, reducing the throttle steam temperature will reduce the rate of damage accumulation. This knowledge is used for fuzzy controller synthesis that is summarized below from the perspective of damage mitigation in the main steam header wall.

\section{A. Fuzzy Rule Base}

The first step in creating the fuzzy rule base is to define universes of discourse and membership functions for the inputs and outputs of the fuzzy controller. Two universes of discourse ( $X_{1}$ and $X_{2}$ ) need to be defined: one for (natural logarithm) of damage rate and the other for the ramp rate of the throttle steam temperature reference trajectory. These two universes of discourse and the associated sets of membership functions are defined as follows.

Natural Logarithm of Damage Rate: universe of discourse: $\dot{d} \in X_{1}=(-\infty, \infty)$ membership functions: $\dot{\mathbf{D}}=\left\{\dot{D}_{1}, \dot{D}_{2}, \ldots, \dot{D}_{n}\right\}$ where $\dot{D}_{i}: X_{1} \rightarrow[0,1] \forall i \in\left\{1, \ldots, n_{1}\right\}$.

Ramp Rate:

universe of discourse: $r r \in X_{2}=\left[R R_{\min }, R R_{\max }\right]$

membership functions: $\mathbf{R R}=\left\{R R_{1}, R R_{2}, \ldots, R R_{n}\right\}$ where $R R: X_{2} \rightarrow[0,1] \forall i \in\left\{1, \ldots, n_{2}\right\}$.

The extreme points $R R_{\min }$ and $R R_{\max }$ are design variables which represent the minimum and maximum allowable ramp rates and the positive integers $n_{1}$ and $n_{2}$ are design variables representing the cardinalities of the damage rate and ramp rate 


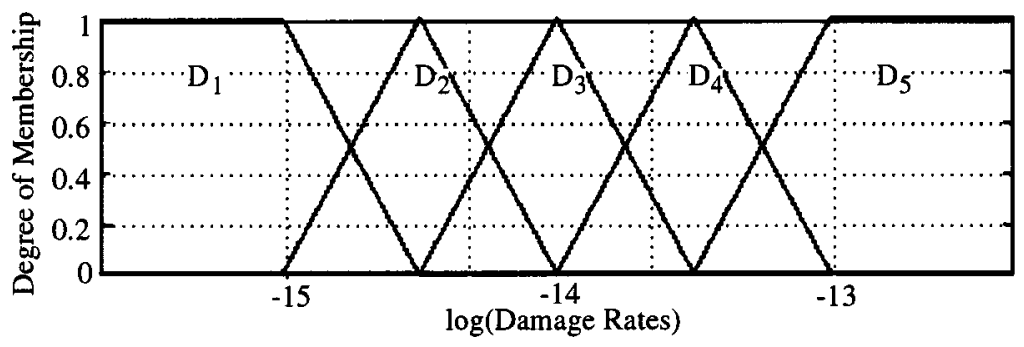

Fig. 5. Damage rate membership functions.

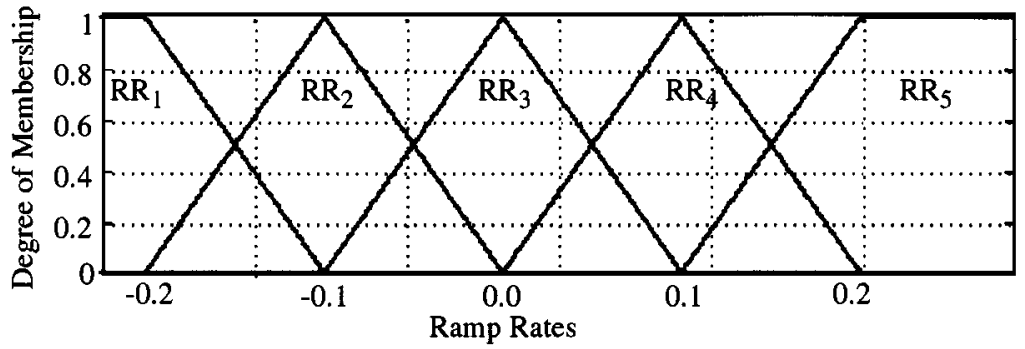

Fig. 6. Ramp rate membership functions.

membership function sets, respectively. If we select $n_{1}=n_{2}=$ $n$, then the set of fuzzy control rules can be conveniently formulated as

$$
\text { Rule } k: \text { If } \dot{D}_{k} \text { then } R R_{k} \quad \forall k \in\{1, \ldots, n\} .
$$

In general, the experience of a human operator needs to be captured and stored in the above rules. The lower bound of the ramp rate is chosen to be $R R_{\min }=-0.2^{\circ} \mathrm{F} / \mathrm{s}\left(-0.11^{\circ} \mathrm{C} / \mathrm{s}\right)$ and the upper bound as $R R_{\max }=+0.2^{\circ} \mathrm{F} / \mathrm{s}\left(+0.11^{\circ} \mathrm{C} / \mathrm{s}\right)$. For the current design, with $n=5$, the $\dot{D}_{i}$ 's are represented as:

$$
\begin{aligned}
& \dot{D}_{1}=\text { very low damage rate } \\
& \dot{D}_{2}=\text { low damage rate } \\
& \dot{D}_{3}=\text { moderate damage rate } \\
& \dot{D}_{4}=\text { high damage rate } \\
& \dot{D}_{5}=\text { very high damage rate }
\end{aligned}
$$

The plots of five damage rate membership functions and five ramp rate membership functions are shown in Figs. 5 and 6, respectively. All membership functions are chosen to be triangle functions that cross each other at the membership value of 0.5. In general, any typical membership function shapes (e.g., Gaussian) can be used.

\section{B. Fuzzy Inputs and Fuzzifier}

The inputs to the inference mechanism of the fuzzy controller are fuzzy sets representing the current measure of damage rate and performance. If information about the measure of damage is known in the form of a nonstationary probability density function (pdf) [8], [7], then the fuzzy input $\dot{D}: X_{1} \rightarrow[0,1]$ can be constructed from this pdf where $\dot{D}(\dot{d})$ is likely to be large at those $\dot{d} \in X_{1}$ that are close to the mean value of the current damage rate. Since parameters for the stochastic damage model of the material, used in the main steam header, are not currently available, the fuzzy control law is designed based on a deterministic (i.e., mean value) model of fatigue damage. Consequently, we have used a fuzzy singleton defined as

$$
\dot{D}(\dot{d})= \begin{cases}1 & \text { when } \dot{d}=\text { estimated mean } \overline{\dot{d}} \\ 0 & \text { otherwise }\end{cases}
$$

The fuzzy singleton in (6) should be replaced by an appropriate function of the damage pdf as it becomes available.

\section{Inference Mechanism and Defuzzifier}

The first phase of the decision process in the inference mechanism is the matching stage whose role is to determine the applicability of each fuzzy control rule to the present set of fuzzy inputs. To this end, the function $\lambda_{i}(D, P)$ is defined for each of the $n$ fuzzy control rules

$$
\lambda_{i}=\max _{\dot{d} \in X_{1}}\left[\min \left(\dot{D}(\dot{d}), \dot{D}^{i}(\dot{d})\right)\right] \quad \forall i \in\{1, \ldots, n\} .
$$

Note that (7) is completely general in the sense that the fuzzy input, $\dot{D}$, is not necessarily a fuzzy singleton defined in (6). However, for a fuzzy singleton, (7) reduces to $\lambda_{i}=\dot{D}^{i}(\dot{\bar{d}})$ where $\bar{d}$ is the estimated mean of of damage rate. The $\lambda_{i}$ 's have a simple interpretation: $\lambda_{i} \in[0,1]$ represents to what extent the current damage rate satisfies the "if" part (or antecedent) of the $i$ th fuzzy control rule. If $\lambda_{i}$ is large, then the $i$ th rule should have a large role in determining the fuzzy controller output.

The second phase of the decision process in the inference mechanism is the summarizing stage whose role is to combine the $\lambda_{i}$ 's defined above with the respective membership functions to produce a fuzzy controller output that is a deterministic quantity. A computationally efficient method to accomplish this task is presented below.

The first step is off-line identification of the center of gravity, $\overline{r r}_{i}$, for the $i$ th membership function, $\Re \Re_{i}, i \in\{1,2, \ldots, n\}$. For the set of ramp rate membership functions chosen in Fig. 


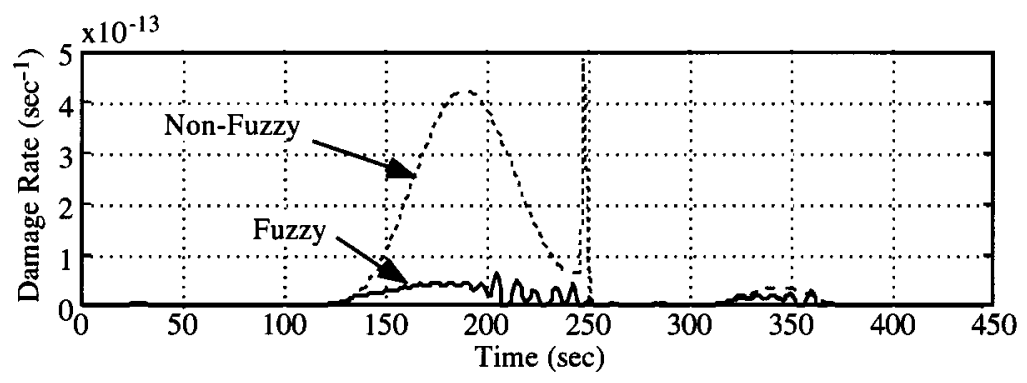

Fig. 7. Fatigue Damage Rate.

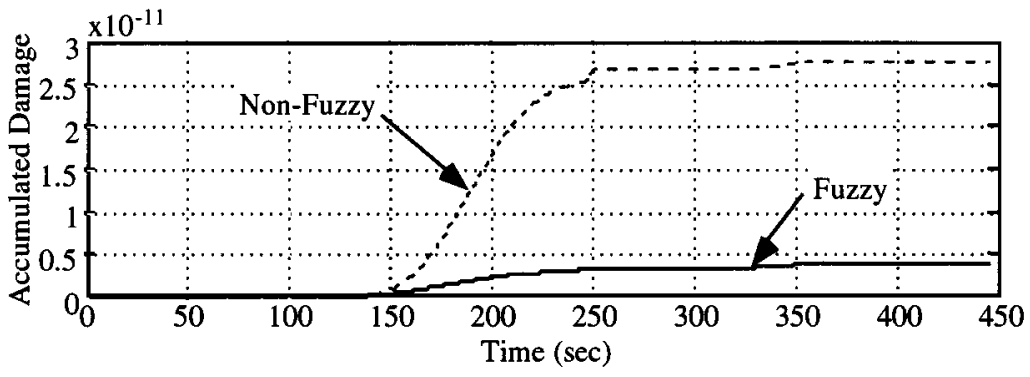

Fig. 8. Fatigue damage accumulation.

6 , the center of gravity of the membership function is chosen based on the following rule:

$$
\begin{aligned}
& \text { If } \Re \Re_{i}=R R_{1}, \quad \text { then } \overline{r r}_{i}=R R_{\min } \\
& \text { If } \Re \Re_{i}=R R_{n}, \quad \text { then } \overline{r r}_{i}=R R_{\max } \\
& \text { If } \Re \Re_{i}=R R_{j}, \quad j=2, \ldots, n-1, \quad \text { then } \overline{r r}_{i} \in X_{2}
\end{aligned}
$$

such that $R R_{j}\left(\overline{r r}_{i}\right)=1$.

In the summarizing stage, the fuzzy controller output is generated a (linear) weighted average of all $n$ fuzzy control rules

$$
r r=\frac{\lambda_{1} \overline{r r}_{1}+\lambda_{2} \overline{r r}_{2}+\cdots+\lambda_{n} \overline{r r}_{n}}{\lambda_{1}+\lambda_{2}+\cdots+\lambda_{n}} .
$$

Note that (10) uses only the centers of gravity of the $R R_{i}$ 's but not their shapes.

\section{SimUlation RESUlTS AND DISCUSSION}

This section presents the results of simulation experiments. In this application, the fuzzy control law presented in Section V is realized in a very simple form

$$
R R(k)= \begin{cases}+0.2 & \text { if } \dot{D}(k) \in(-\infty,-15] \\ -0.2 \dot{D}(k)-2.8 & \text { if } \dot{D}(k) \in(-15,-13) \\ -0.2 & \text { if } \dot{D}(k) \in[-13, \infty)\end{cases}
$$

The rationale for performing all of the steps in Section $\mathrm{V}$ instead of directly creating a simple realization in (10) is that it is more intuitive to view the design through the fuzzy controller framework. Also, it is more convenient to modify the current design by following the procedure in Section V. The procedure of fuzzy damage controller design in Section $\mathrm{V}$ is applicable to other plant components for different types of damage. The fuzzy damage controller could be more complex for other applications.

Figs. 7-10 compare the trajectories of the four power plant outputs with and without a fuzzy controller in place where the robust linear tracking control in Fig. 1 is identical for both configurations. In the sequel, the configuration without fuzzy control is called nonfuzzy, which retains the damage-mitigating features of the linear tracking controller as discussed in Section IV. In the nonfuzzy control configuration, $y^{\text {dyn }}$ does not exist and $y^{\text {reg }}$ consists of the all four output variables (see Fig. 1). In the fuzzy control configuration, $y^{\text {dyn }}$ consists of throttle steam temperature and the vector signal $y^{\text {reg }}$ consists of the remaining three output variables, hot reheat steam temperature, throttle steam pressure, and electric power. Therefore, the reference signal $y^{\text {ref }}$ for throttle steam temperature is modified on-line by the fuzzy damage controller while and the vector reference signal $y^{\text {set }}$ consisting of the remaining three output variables is not modified.

Simulation experiments have been constructed based on the fact that large change in load demand may occur within several minutes. Typically, a loading following fossil plant may experience rapid load maneuvering within a few minutes during late evenings and early mornings especially if this plant supports a number of base-load units. The following scenario of rapid load maneuvering is simulated in this paper.

- The electrical power is increased from $210 \mathrm{MWe}(40 \%$ load) to 525 MWe (100\% load) over a period of $240 \mathrm{~s}$ to show efficacy of the fuzzy controller. The plant operation requirements are to maintain the damage-causing variables within the following bounds.

- The throttle steam temperature at $950^{\circ} \mathrm{F} \pm$ $10^{\circ} \mathrm{F}\left(510^{\circ} \mathrm{C} \pm 5.56^{\circ} \mathrm{C}\right)$.

- The hot reheat steam temperature at $1000^{\circ} \mathrm{F} \pm$ $15^{\circ} \mathrm{F}\left(537.8^{\circ} \mathrm{C} \pm 8.33^{\circ} \mathrm{C}\right)$.

- The throttle steam pressure at $2415 \mathrm{psi} \pm 100 \mathrm{psi}$ (16.65 $\mathrm{MPa} \pm 0.689 \mathrm{MPa})$.

Figs. 7 and 8 compare the fatigue damage rate and accumulation, respectively, under nonfuzzy control with those under fuzzy control that manipulates the throttle steam temperature 


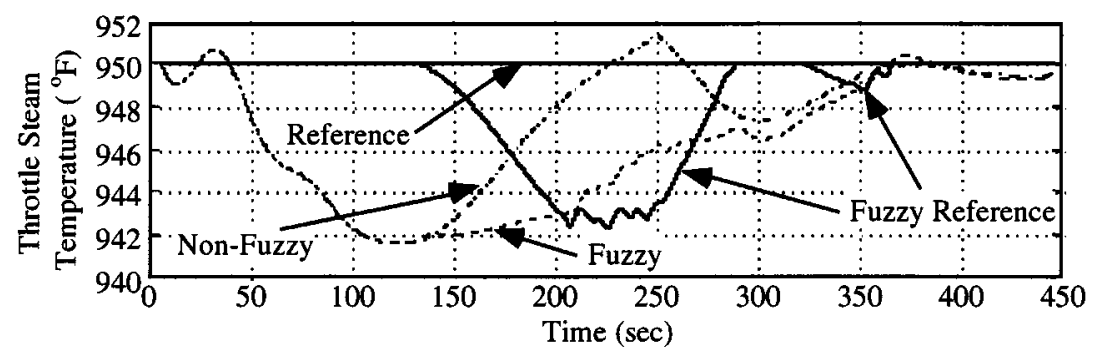

Fig. 9. Throttle steam temperature transients.

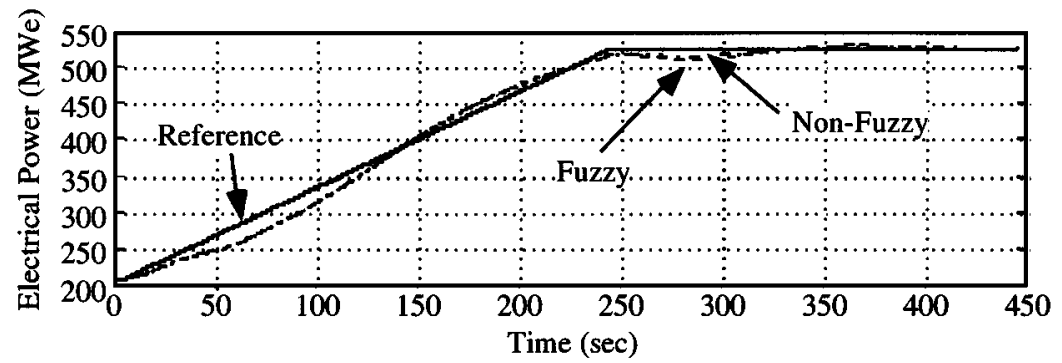

Fig. 10. Electrical power transients.

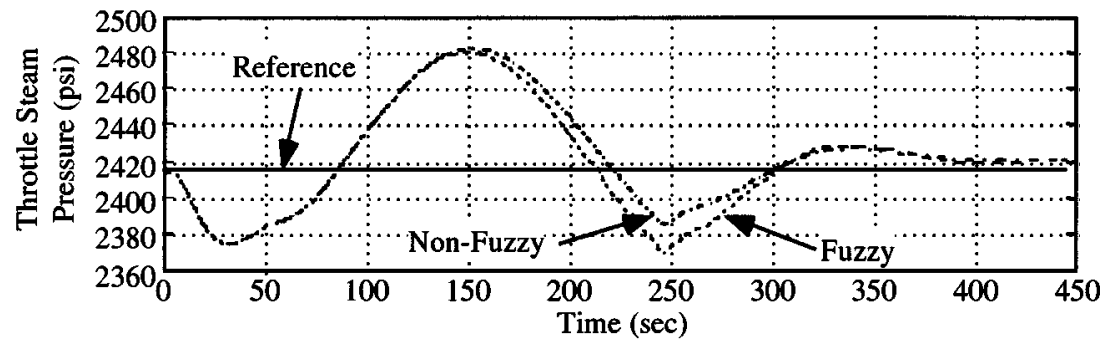

Fig. 11. Throttle steam pressure transients.

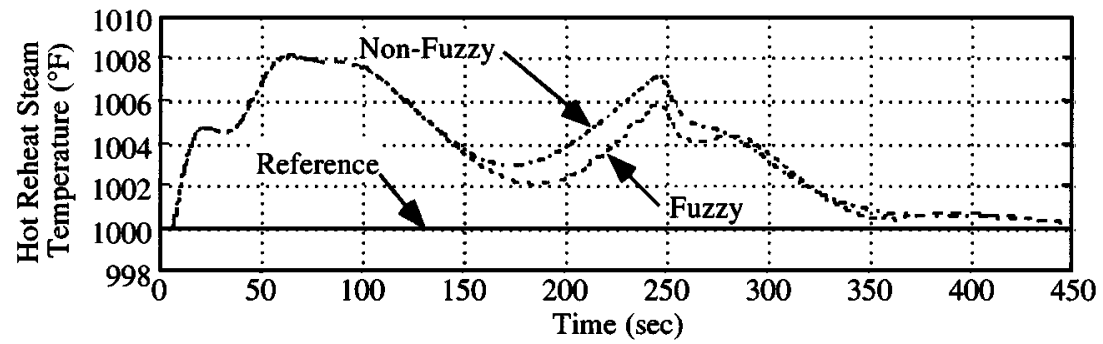

Fig. 12. Hot reheat steam temperature.

as seen in Fig. 9. Fig. 8 shows that the fuzzy damage controller yields approximately a seven-fold saving in the incremental fatigue life of the main steam header as compared to the nonfuzzy controller while the loss of performance due to tracking error in generated power (Fig. 10) is insignificant during this load ramp-up maneuver. Similar gains in incremental fatigue life (not shown in this paper) are also obtained during load ramp down operations. The reason for this significant gain in fatigue damage reduction is that the fuzzy controller deliberately manipulates the reference signal of throttle steam temperature (Fig. 9) to reduce the thermal stresses (and hence thermomechanical fatigue damage) in the main steam header. The actions of the fuzzy controller affect the dynamics of all four output variables in this multivariable system. The mild degradation in tracking of throttle steam pressure (Fig. 11) has negligible bearing on the plant performance. The fuzzy controller also improves the response of hot reheat steam temperature (Fig. 12) that enhances structural durability of the hot reheat steam header. Kallappa and Ray [6] have quantified the net gain as a combined measure of plant performance in terms of load tracking, and structural durability in terms of damage in several plant components.

\section{SUMMARY AND CONCLUSION}

This paper presents a procedure for synthesizing a damage mitigating control system for processes where structural integrity is an important issue. The proposed control system has a two-tier structure. A linear sampled-data controller 
tracks a reference trajectory vector in the lower tier while a fuzzy-logic-based damage controller at the upper tier makes a tradeoff between system performance and damage in critical components. The synthesis procedure is demonstrated by simulation experiments on the model of a fossil power plant. The simulation results demonstrate the feasibility of simultaneously regulating plant dynamic performance and structural damage in a real-time setting based on the available measurements. Since the fuzzy controller makes the decision of changing the pressure reference signal based on on-line damage predictions, it can react to unexpected situations, such as damage caused by an external disturbance, that cannot be predicted a priori.

The concept of damage mitigating control as described in this paper is of significant engineering importance. For example, including damage in the control scheme leads to the possibility of extending the service life of the controlled process as well as increasing the mean time between major maintenance actions. During a particular mission, if the damage of a critical component exceeds the expected level, it may be possible to modify the operation of the plant on-line so that the current mission can be completed with an acceptable amount of damage. The tradeoff is a (possibly small) reduction in the system performance. Changing the operation of the plant in this situation may also prevent a potentially dangerous situation caused by the failure of a critical plant component. Therefore, the damage mitigating control has the potential of providing both economic benefits and enhancement of operational safety.

\section{REFERENCES}

[1] G. J. Balas, J. C. Doyle, K. Glover, A. Packard, and R. Smith, $\mu$-Analysis and Synthesis Toolbox: MUSYN Inc. and The Math Works, Inc., 1993.

[2] B. A. Bamieh and J. B. Pearson Jr., "A general framework for linear periodic systems with applications to $H_{\infty}$ sampled-data control," IEEE Trans. Automat. Contr., vol. 37, pp. 418-435, Apr. 1992.

[3] X. Dai and A. Ray, "Damage-mitigating control of a reusable rocket engine: Parts I and II," ASME J. Dyn. Syst.s, Measurement, Contr., vol. 118, no. 3, pp. 401-415, Sept. 1996.

[4] M. S. Holmes and A. Ray, "Fuzzy damage-mitigating control of mechanical structures," ASME J. Dyn. Syst., Measurement. Contr., vol. 120, no. 2, pp. 249-256, June 1998.

[5] P. Kallappa, M. S. Holmes, and A. Ray, "Life extending control of fossil power plants for structural durability and performance enhancement," Automatica, vol. 33, no. 6, pp. 1101-1118, 1997.

[6] P. Kallappa and A. Ray, "Fuzzy wide range control of fossil power plants for life extension and robust performance," Automatica, vol. 36, no. 1, pp. 69-82, 2000.
[7] A. Ray, "Stochastic modeling of fatigue crack damage for risk analysis and remaining life prediction," ASME J. Dyn. Syst., Measurement, Contr., vol. 121, no. 3, pp. 386-393, Sept. 1999.

[8] A. Ray and S. Phoha, "Stochastic modeling of fatigue crack damage for information-based maintenance," Ann. Oper. Res., vol. 91, pp. 191-204, 1999.

[9] A. Ray, M.-K. Wu, M. Carpino, and C. F. Lorenzo, "Damage-mitigating control of mechanical systems: Parts I and II," ASME J. Dyn. Syst., Measurement. Contr., vol. 116, no. 3, pp. 437-455, Sept. 1994.

[10] S. C. Stultz and J. B. Kitto, Eds., Steam/Its Generation and Use, 40th ed. Barberton, OH: Babcock \& Wilcox, 1992.

[11] C.-K. Weng, A. Ray, and X. Dai, "Modeling of power plant dyanmics and uncertainties for robust control synthesis," Appl. Math Modeling, vol. 20, pp. 501-512, July 1996.

[12] C.-K. Weng and A. Ray, "Robust wide-range control of steam-electric power plants," IEEE Trans. Contr. Syst. Technol., vol. 5, pp. 74-88, Jan. 1997.

[13] H. Zhang and A. Ray, "Robust damage-mitigating control of mechanical systems: Experimental validation on a test apparatus," ASME J. Dyn Syst., Measurement, Contr., vol. 121, no. 3, pp. 377-385, Sept. 1999.

Michael Holmes received B.S.E.E., M.S.E.E., and Ph.D. degrees in electrical engineering with minor in mechanical engineering from the Pennsylvania State University, University Park, PA, in 1991, 1994, and 1997, respectively.

His area of research was the application of fuzzy and multivariable robust control theory to the damage-mitigating control of mechanical systems. From October 1997 to January 1999, he was with the Raytheon Systems Company. $\mathrm{He}$ is currently a Senior Member of the Engineering Staff at Lockheed Martin Naval Electronics and Surveillance Systems-Surface Systems, Moorestown, NJ.

Asok Ray (SM'83) earned the Ph.D. degree in mechanical engineering from Northeastern University, Boston, MA in 1976.

He joined the Pennsylvania State University, University Park, in July 1985 , and is currently a Professor of Mechanical Engineering, a Graduate Faculty in the Inter-College Program in Materials, and a Graduate Faculty of Electrical Engineering. Prior to joining Penn State, he held research and academic positions at Massachusetts Institute of Technology, Cambridge, and Carnegie Mellon University, Pittsburgh, PA, as well as research and management positions at GTE Strategic Systems Division, Charles Stark Draper Laboratory, and MITRE Corporation. He has authored or coauthored 300 research publications including about 150 scholarly articles in refereed journals and research monographs.

Dr. Ray is a Fellow of ASME and an Associate Fellow of AIAA. He is an Editor of the IEEE TRANSACTIONS ON AEROSPACE AND ELECTRONIC SYSTEMS and an Associate Editor of the IEEE TRANSACTIONS ON CONTROL SySTEMS TECHNOLOGY. He had been an Associate Editor of the ASME Journal of Dynamic Systems, Measurement, and Control. 